\title{
actualización
}

\section{Emergencias y urgencias en Reumatología clínica}

\author{
Alfredo S. Arturi, Valeria Arturi, Delfor Giacomone \\ Cátedra de Post-grado de Reumatología, Facultad de Ciencia Médicas, Universidad Nacional de La Plata.
}

\section{Palabras clave:}

emergencias, urgencias, reumatología, enfermedades autoinmunes.

\section{R E S U M E N}

En la presente revisión se analizan los casos de Urgencias y Emergencias en Reumatología y Enfermedades Autoinmunes Sistémicas del adulto y juveniles; las mismas son situaciones que requieren asistencia médica inmediata. Además se mencionan las complicaciones agudas que surgen de las mismas (infección, desorden metabólico, insuficiencia de órgano) y las reacciones adversas que pueden surgir de los tratamientos efectuados. Se destacan las patologías por órgano o sistema y luego por síndrome o enfermedad. La revisión tiene la intención de ser de ayuda al médico joven para un rápido reconocimiento, diagnóstico y tratamiento de las patologías agudas y sus complicaciones en la Reumatología actual.

\section{A B S T R A C T}

In the present review, cases of Urgencies \& Emergencies in Rheumatology and Systemic Autoimmune Diseases of adults and juveniles patients are analyzed; these are situations that require immediate medical attention. In addition, acute complications arising from background diseases (infection, metabolic disorder, organ failure) and adverse events that may arise from treatments performed for this purpose are mentioned. Pathologies by organ or system and then by syndrome or disease are highlighted. This review is intended to be helpful for young physicians in order to perform rapid recognition, diagnosis and treatment of acute pathologies and their complications in the current Rheumatology practice. 
La Reumatología es actualmente una especialidad médica que incluye innumerables afecciones, dentro de ellas, un importante capítulo lo constituyen las Enfermedades Autoinmunes Sistémicas (EAS). Los adelantos ocurridos desde el punto de vista etiopatogénico, clínico, de métodos complementarios de diagnóstico y terapéuticos han permitido realizar diagnósticos tempranos y tratamientos más efectivos.

Si bien es reconocida, en la especialidad, la mayor frecuencia de enfermedades crónicas, en esta revisión se pondrá énfasis en aquellas afecciones en la que las emergencias y las urgencias médicas son relevantes.

El término Emergencia es definido por la AMA (American Medical Association) como aquellas situaciones en que se juega la vida del paciente o la función de un órgano en forma inmediata. Por otro lado la Urgencia es la situación en la cual el paciente o su familia o alguien responsable requieren inmediato cuidado de la salud aunque no sea necesario comprometer la vida. Por otro lado, la WHO (World Health Organization) se refiere a Emergencia cuando está en juego la vida del paciente en pocos minutos y requiere atención inmediata ${ }^{1,2}$. De acuerdo a ello y como ejemplo lo es el síndrome antifosfolípido (SAF) catastrófico que es una Emergencia en que está comprometida la vida del paciente en forma aguda, mientras que el de Urgencia es aquella en la que se debe actuar también rápidamente pero no corre riesgo la vida, ejemplo característico de ello es el ataque agudo de gota.

En una revisión realizada por Gutiérrez González LA y cols., en 2015, el autor analiza las emergencias reumatológicas en casos de SAF catastrófico, síndrome pulmón-riñón, vasculitis del $\mathrm{SNC}$, síndrome anti-Ro (lupus neonatal), síndrome de activación macrofágica, crisis renal esclerodérmica, artritis séptica y subluxación atlantoaxoidea ${ }^{3}$

En un brief report de Bellan M y cols., en 2016, se analizaron los eventos musculoesqueléticos no traumáticos que a menudo podrían comprometer la vida (EMNT) en un centro de Emergencias. Definió Emergencia a las condiciones con riesgo de alta morbimortalidad, mientras que Urgencias a aquellas que requieren una rápida consulta al especialista. De 54.915 pacientes evaluados en 1 año, 1652 (3\%) padecieron EMNT; 2/708 (0,3\%) correspondieron a emergencias y $210 / 708(29,7 \%)$ a urgencias. Concluyen que las emergencias musculoesqueléticas son relativamente poco frecuentes en un centro de Emergencia ${ }^{4}$.

En 2016, Guillen Astete C y cols. analizaron la prevalencia de afecciones musculoesqueléticas no traumáticas como motivo de consulta en un centro de Emergencias. Analizaron retrospectivamente 2000 historias clínicas electrónicas. La prevalencia de EMNT fue alta, del 13,8\%; de ellas, el motivo más frecuente fue la lumbalgia, le siguieron compromiso de rodilla, cadera, esqueleto axial cervical, hombro, mano y pie ${ }^{5}$. El mismo autor, en 2017, analiza 1 año de experiencia en una Unidad de Urgencias Reumatológicas y Musculoesqueléticas; tuvieron 1788 atenciones en 1663 pacientes; la edad media de los pacientes fue de 67 años; 633 (35,4\%) correspondieron a procesos inflamatorios $y$ el resto $(64,6 \%)$ a procesos mecánicos, degenerativos, periarticulares y partes blandas. Los motivos de consulta más frecuentes fueron los relacionados a la articulación de la rodilla, región lumbar y hombro en ese orden ${ }^{6}$.

En la presente revisión nos hemos propuesto analizar en forma amplia las Emergencias o Urgencias en Reumatología ${ }^{1}$.

En un principio se clasificarán las situaciones de acuerdo al tipo de la Emergencia y Urgencia (motivo de consulta); posteriormente se hace un análisis diferenciado de cada caso en particular y por último se analizan por patología ${ }^{4,7}$.

A nuestro juicio, para la atención médica de este tipo de patologías se requiere:

- Amplio conocimiento de Clínica Médica y Reumatología por parte del profesional.

- Centro asistencial de alta complejidad, y/o

- Grupo multidisciplinario de profesionales que pueda colaborar en forma activa con el médico a cargo del paciente.
Los signos y síntomas que pueden ser motivo de presentación de una Urgencia y Emergencia en Reumatología son:

- Fiebre de reciente comienzo

- Compromiso Pleuropulmonar

- Enfermedad pulmonar aguda

- Hemoptisis

- Disnea aguda

- Crisis asmática

- Pleuritis

- Neumonitis

- Hemorragia pulmonar

- Alteraciones Gastrointestinales

- Hematemesis

- Melena

- Abdomen agudo médico o quirúrgico

- Perforación intestinal

- Pancreatitis

- Afección Cardiovascular

- Precordialgia

- Pericarditis

- Miocarditis

- Endocarditis

- Crisis hipertensiva

- Hipotensión severa

- Aneurismas

- Compromiso Oftalmológico

- Pérdida de visión aguda

- Diplopía

- Ojo rojo inflamatorio

- Escleromalacia perforans

- Alteración Renal

- Insuficiencia renal aguda rápidamente progresiva

- Cólico renal

- Glomerulonefritis aguda

- Crisis renal hipertensiva

- Compromiso Neuropsiquiátrico

- Parálisis de nervio periférico

- Pérdida de fuerza muscular

- Dolor radicular agudo

- Accidente vascular cerebral

- Isquemia periférica con o sin necrosis digital

- Mielitis transversa

- Alteración cognitiva aguda

- Psicosis

- Convulsiones

- Cefalea aguda

- Trastorno psiquiátrico agudo

- Vértigo

- Alteraciones Hematológicas/Vasculares

- Hematomas espontáneos

- Anemia aguda (hemolítica, pérdida masiva)

- Trombosis venosa/tromboembolismo pulmonar (TEP)

- Accidente vascular cerebral (ACV)

- Infarto agudo de miocardio (IAM)

- Ruptura de aneurismas

- Lesiones Cutaneomucosas

- Rash cutáneo extendido

- Vasculitis cutánea

- Necrosis de piel, mucosas, tejido subcutáneo

- Isquemia digital

- Alteraciones vinculadas a Ortopedia y Traumatología

- Fractura por osteoporosis

- Luxación articular periférica

- Luxación atlanto-axoidea

- Compromiso Articular

- Monoartritis aguda

- Oligo o poliartritis aguda

- Desorden Endocrino-metabólico

- Diabetes descompensada

- Insuficiencia adrenal

- Infección aguda 
- Artritis reactiva

- Artritis séptica

- Espondilodiscitis séptica

- Infección sistémica

- Síndrome de activación macrofágica

A grandes rasgos, intentamos clasificar en forma diferenciada ejemplos relevantes por su frecuencia de Emergencias; las mismas son:

- ACV isquémico o hemorrágico

- Crisis hipertensiva

- Pericarditis constrictiva, endocarditis aguda, miocarditis e insuficiencia cardíaca

- Neumonitis lúpica aguda con o sin hemorragia pulmonar

- Púrpura trombocitopénica aguda

- Anemia hemolítica

- Convulsiones

- Parálisis neurológica periférica

- Diabetes descompensada

- Infección severa

- Fractura de raquis o cadera

- Luxación atlanto-axoidea con compresión de médula espinal

- Amaurosis aguda

Por otro lado, los ejemplos más frecuentes de las Urgencias son:

- Pleuritis, pericarditis

- Ataque cristálico agudo: gota, pseudogota

- Radiculopatía compresiva a nivel axial y/o periférica

- Infecciones no graves

- Fracturas

- Fenómeno de Raynaud o isquemia periférica

Entendemos que esta clasificación es meramente especulativa, pues existen grandes variaciones en cada caso en particular.

Otro punto importante a considerar es tener en cuenta las secuelas que suelen quedar de episodios, por lo que ameritan a actuar con rapidez para evitar situaciones que pueden ser importantes, como por ejemplo:

- ACV u otra lesión neurológica

- IAM u otra isquemia periférica

- Insuficiencia renal crónica

- Amaurosis u otra lesión ocular definitiva

- Insuficiencia respiratoria crónica

- Atribuible a infecciones

Un caso particular, por su frecuencia y por las innumerables situaciones en las que puede presentarse, es la fiebre que, de hecho, suele ser de diversos tipos (permanente, en picos, vespertina).

Ante un cuadro febril, hay que considerar:

- Brote de LES

- Infecciones intercurrentes: bacterianas (endocarditis),

micóticas, virales

- Enfermedad de Still del adulto

- Vasculitis sistémica

\section{Emergencias/Urgencias por patologías}

Hay que considerar además que cada síndrome o Enfermedad Autoinmune Sistémica suele tener situaciones propias de la afección. Analizamos las Emergencias/Urgencias por patologías, evaluaremos cada una en particular y sobre todo en lo que hay que examinar en cada caso puntual 8,9,10,11,12:

- Lupus Eritematoso Sistémico (LES)

- Síndrome Antifosfolípido (SAF)

- Artritis Reumatoidea (AR)

- Esclerodermia (ED)

- Miopatías Inflamatorias Idiopáticas (MI)

- Vasculitis

- Monoartritis
- Oligo/Poliartritis

- Ortopedia y Traumatología

- Complicación de tratamientos

\section{Lupus Eritematoso Sistémico}

Paradigma de Enfermedad Autoinmune Sistémica, enfermedad que predomina en la mujer en la vida fértil; la hiperreactividad de las células $B$ se traduce en formación de autoanticuerpos patogénicos, los mismos pueden afectar variados órganos y sistemas en forma coincidente o aditiva. Es por ello que es la enfermedad que nos ofrece la mayor cantidad de eventos emergentes, de ellos los más importantes son ${ }^{13,14}$ 15,16:

- Pleuritis/pericarditis/miocarditis/endocarditis/fallo valvular cardíaco

- Vasculitis sistémica

- Anemia hemolítica, púrpura trombocitopénica

- Brote cutáneo agudo sistémico, paniculitis, necrosis de tejido

- Afectación neurológica: encefalopatía, ACV, parálisis periférica, estado confusional, psicosis, convulsiones, mielitis transversa, trastornos cognitivos

- Coronariopatía, IAM, crisis hipertensiva, hipertensión pulmonar descompensada

- Amaurosis u otro daño ocular agudo

- Glomerulonefritis, insuficiencia renal aguda, enfermedad vascular renal, nefritis intersticial aguda

- Enfermedad pulmonar aguda: neumonitis lúpica aguda, hemorragia pulmonar

- Hematemesis, melena, vasculitis mesentérica, perforación visceral

- Pancreatitis

- Fractura, colapso vertebral, rupturas tendinosas

- Brote multisistémico severo

El LES es una enfermedad sumamente compleja que además de todos los signos y síntomas que produce per se existen un sinnúmero de comorbilidades que también cursan con emergencias (asociaciones frecuentes); ejemplo de ellas son:

- Síndrome antifosfolípido (SAF), complicaciones

isquémicas/obstétricas

- Infecciones

- Fracturas

- Diabetes descompensada

- Complicaciones de los tratamientos

Otras situaciones que no son emergentes pero deben ser consideradas por su frecuencia y daño son: osteoporosis, dislipemia, hipotiroidismo, depresión, fibromialgia, síndrome de Sjögren secundario, entre otras.

\section{Síndrome Antifosfolipídico}

Es otra de las enfermedades en las cuales los eventos trombóticos agudos severos arteriales o venosos son origen de emergencias; los mismos están relacionados a la presencia de anticuerpos antifosfolípidos (anti-cardiolipinas IgM e IgG, anti- $\beta 2$ GPI IgG e IgM y el inhibidor lúpico). El cuadro, como es bien sabido, puede ser primario o estar asociado a otra enfermedad autoinmune o infecciosa (SAF secundario). Los signos y síntomas asociados al SAF son $9,10,15,17$ :

- Isquemia arterial aguda central o periférica

- Trombosis venosa aguda, TEP

- Vasculitis retinal, amaurosis

- Cefalea incapacitante

- Complicaciones gineco-obstétricas: síndrome HELLP, aborto espontáneo

- SAF catastrófico

\section{Artritis Reumatoidea}

En los últimos años y habida cuenta de los procedimientos de 
diagnóstico, y de hecho el diagnóstico precoz, las otras situaciones de emergencia se han reducido en cantidad; de cualquier manera hay que recordar que la artritis reumatoidea (AR) no controlada puede presentar en cualquier momento de su evolución ${ }^{4,8,12}$ :

- Vasculitis sistémica

- Luxación atlanto-axoidea

- Ruptura quiste de Baker

- Escleromalacia perforante

- Ruptura tendinosa

- Fractura

- Infecciones

\section{Esclerodermia}

La Esclerodermia es una enfermedad crónica del tejido conectivo de causa desconocida, afecta piel, sistema vascular y órganos internos. Existen diferentes tipos de Esclerodermia; dentro de ella, la Esclerosis Sistémica Progresiva es la forma más grave de la enfermedad, en la cual la crisis renal esclerodérmica era una causa de morbimortalidad significativa y que de acuerdo a las terapéuticas actuales se puede prevenir y controlar; de cualquier manera en esta enfermedad se puede observar una diversidad de emergencias incluyendo todas sus formas de la enfermedad ${ }^{9,10}$ :

- Crisis renal esclerodérmica

- Enfermedad pulmonar con insuficiencia respiratoria aguda

- Hipertensión pulmonar descompensada

- Fenómeno de Raynaud severo con necrosis digital

- Vasculitis sistémica

- Enfermedad coronaria, IAM, insuficiencia cardíaca

\section{Miopatías Inflamatorias Idiopáticas (MII)}

Existen innumerables enfermedades musculares, por lo que en este apartado nos ocuparemos exclusivamente de las que tienen componentes básicamente inflamatorio (miopatías inflamatorias idiopáticas, MII) y que pueden cursar con emergencias (excluimos las neuromiopatías, genéticas, mitocondriales). Las MII pueden presentar cuadros emergentes que incluyen $5,6,11,18$ :

- Dermatomiositis

- Polimiositis

- Miositis con cuerpos de inclusión

- Síndrome antisintetasa

- Miositis necrotizante inmunomediada

- Miopatía paraneoplásica

Las MII pueden provocar, o estar asociadas a emergencias y urgencias tales como:

- Cuadro cutáneo extendido

- Vasculitis, perforación intestinal

- Insuficiencia respiratoria aguda

- Miocarditis con insuficiencia cardíaca

- Neumonía por aspiración

- Rabdomiólisis

\section{Vasculitis}

Las vasculitis son un grupo de afecciones heterogéneas que comprometen por definición los vasos sanguíneos de cualquier calibre, provocando estrechamiento, oclusión y necrosis; los síntomas están relacionados estrechamente al sitio y calibre del vaso afectado. La enfermedad inflamatoria vascular puede ser primaria (vasculitis puras), o estar asociadas a otra enfermedad (vasculitis secundaria) $^{10,16,19}$.

Las vasculitis puras sistémicas que con mayor frecuencia presentan cuadros agudos son:

- Poliarteritis nodosa

- Vasculitis ANCA

- C: Granulomatosis con poliangeítis (granulomatosis de Wegener)

- P: Poliangeítis microscópica

- Granulomatosis eosinofílica con poliangeítis

(síndrome de Churg-Strauss)
- Vasculitis por hipersensibilidad

- Arteritis de células gigantes

- Púrpura de Schönlein-Henoch

- Vasculitis crioglobulinémica

La sintomatología vinculada a las vasculitis es muy variada y requiere de una atención especial.

Es de vital importancia conocer el diagnóstico primario del tipo de vasculitis para interpretar con rapidez el cuadro agudo; lo más frecuentes y dependiendo de cada una son:

- Asma

- Hemorragia pulmonar

- Insuficiencia renal aguda

- Infarto agudo de miocardio

- ACV - síndromes neurológicos periféricos

- Necrosis distal

- Vasculitis mesentérica

- Perforación de víscera hueca

- Amaurosis aguda

Un caso particular es el síndrome pulmón - riñón de causa autoinmune, en el cual el compromiso del parénquima pulmonar y renal se encuentren afectadas en forma simultánea y con diferente gravedad. Las causas más frecuentes de este síndrome son ${ }^{16,19}$.

- Vasculitis ANCA: Poliangeítis microscópica

Vasculitis granulomatosa (Wegener)

- Enfermedad de Goodpasture

- LES

Existen otro sinnúmero de afecciones no inmunes que pueden comprometer ambos órganos en forma simultánea o con poco período de tiempo que no son tratados en esta revisión.

\section{Monoartritis aguda}

La monoartritis es el proceso mediante el cual se produce una inflamación articular en una sola articulación; la misma puede ser aguda, subaguda o crónica. Dentro de las formas agudas hay que considerar $2,3,8,20,21,22$ :

- Sinovitis microcristalinas: gota (urato monosódico) pseudogota (pirofosfato de calcio)

seudo-pseudogota (fosfatos cálcicos básicos)

- Artritis séptica: nativa o protésica

- Artritis/tenosinovitis psoriásica

- Síndrome R3S PE

\section{Oligo-poliartritis}

El compromiso de más de una articulación afectada en forma coincidente con un proceso inflamatorio agudo puede corresponder a una diversidad de patologías. Las oligo y poliatritis que pueden cursar con cuadros agudos son: $2,3,8,20,21,22$

- AR

- Microcristalinas

- Gota

- Pseudogota

- Pseudo-pseudogota

- Artritis psoriásica

- Síndrome R3S PE

\section{Infecciones}

Un tema relevante en Reumatología es el de las infecciones oportunistas, las mismas pueden ser agudas y severas; la mayoría de las enfermedades autoinmunes cursan con inmunodepresión, a ello hay que agregarle alguna participación de los tratamientos utilizados en estas patologías.

Ante un cuadro agudo, siempre debe pensarse y descartar infección intercurrente; las mismas son lo más frecuente en el contexto general. Las infecciones pueden ser:3,8,12,22

\section{A-Sistémicas}

- Enfermedad bacteriana sistémica en organismo inmunocomprometido 
- Virales

- Micóticas

- Vinculada a los tratamientos: corticoesteroides, inmunosupresores, terapias biológicas, infiltraciones, cirugías

B-Articulares

- Artritis séptica primaria de la articulación

- Artritis séptica post-punción

- Artritis séptica protésica

- Artritis séptica: estafilococo, gonococo, TBC

- Artritis reactiva: urogenital, enteral

- Fiebre reumática

\section{Emergencias vinculadas a la Ortopedia y Traumatología}

Con nuestros colegas ortopedistas y traumatólogos compartimos enfermedades o situaciones en las cuales debemos actuar en conjunto. Es de mucha importancia el enfoque compartido ante ${ }^{4,18,23,24,25 \text {. }}$

- Fracturas: periférica colapso vertebral

- Luxaciones articulares o protésicas

- Ruptura de quiste de Becker

- Compresiones radiculares cervicales (cervicobraquialgia)

- Compresiones radiculares lumbares (lumbociatalgia)

- Artritis traumática

\section{Tratamientos}

Innumerables emergencias médicas y/o quirúrgicas están relacionadas a los tratamientos instituidos; los mismos deben ser cuidadosamente evaluados porque en ocasiones pueden ser más graves que la enfermedad en la cual se indicó. Las drogas son de lo más variadas e incluyen AINE, corticoesteroides, inmunorreguladores, inmunodepresores, terapias biológicas, plasmaféresis, entre otras.

Las comorbilidades habitualmente existentes en los pacientes complican la situación (polipatologías con polifarmacia), tal es el caso de diabetes, HTA, hipotiroidismo, ansiedad, depresión; de tal manera que pueden ocurrir manifestaciones vinculadas a los tratamientos ${ }^{2,12}$ :

- Digestivas

- Infecciosas

- Hepáticas

- Renales

- Oculares

- Metabólicas (diabetes)

- Osteoporosis (fracturas)

\section{Emergencias en Reumatología Pediátrica}

Aunque las emergencias médicas son poco frecuentes en las enfermedades reumáticas pediátricas, hay situaciones en las que su pronto reconocimiento y tratamiento son esenciales para minimizar la morbilidad y la mortalidad de estos cuadros. Estas complicaciones pueden ocurrir en el marco de una patología preexistente, pero también pueden ser su síntoma de presentación, por lo tanto, el clínico pediatra debe estar capacitado para su rápido reconocimiento.

En esta sección, haremos mención de entidades que generalmente son propias de la edad pediátrica, ya que las complicaciones de otras patologías comunes a la población adulta han sido descriptas precedentemente (artritis séptica, LES, SAF).

\section{Artritis idiopática juvenil sistémica (AIJs) - Enfermedad de Still del adulto}

La AIJs se caracteriza por la presencia de fiebre en picos de $39^{\circ}$ o más, 1-2 por día, vespertinos o nocturnos, durante dos o más semanas (puede preceder a la artritis en semanas o meses, lo que dificulta muchas veces el diagnóstico). Rash cutáneo máculo- eritematoso, de color rojo asalmonado, localizado en tronco y raíz de miembros evanescente y generalmente se encuentra asociado a los picos febriles. Adenomegalias generalizadas, hepatoesplenomegalia y poliserositis son frecuentes y forman parte del cuadro clínico ${ }^{26}$.

El diagnóstico diferencial debe efectuarse fundamentalmente con enfermedades oncohematológicas (leucemia), procesos infecciosos, otras enfermedades autoinmunes sistémicas y síndromes autoinflamatorios.

La enfermedad de Still del adulto es considerada por muchos autores como parte del espectro de AIJs de inicio en edad más avanzada ${ }^{27}$. Presenta manifestaciones clínicas similares, aunque no es indispensable la presencia de artritis para su diagnóstico (Criterios de Yamaguchi) ${ }^{28}$.

\section{Síndrome de activación macrofágica (SAM)}

Una de las complicaciones más severa de la AIJs y de la enfermedad de Still del adulto lo constituye el síndrome de activación macrofágica (SAM), que puede observarse también como complicación del LES y la enfermedad de Kawasaki. Se manifiesta clínicamente entre el $7 \%$ y el $15 \%$ de los pacientes, aunque hay trabajos que describen hasta un $50 \%$ de SAM oculto o subclínico. La mayoría de los casos ocultos se diagnostican por una biopsia de médula efectuada por sospecha de enfermedad hematológica ${ }^{29}$.

El SAM puede ocurrir espontáneamente como una complicación del proceso inflamatorio subyacente o ser desencadenado por una infección, un cambio en la terapia con fármacos o un efecto tóxico de un medicamento.

Manifestaciones clínicas características son la alteración del estado general, fiebre alta persistente, hepatoesplenomegalia, linfadenopatía generalizada, disfunción hepática, alteraciones neurológicas y coagulopatía.

Las alteraciones de laboratorio características son la pancitopenia, el aumento de ferritina, triglicéridos, enzimas hepáticas, dímero $\mathrm{D}, \mathrm{LDH}$ y la disminución de fibrinógeno. En médula ósea se observan macrófagos con actividad hemofagocítica.

El siguiente cuadro puede ayudar a efectuar el diagnóstico diferencial entre SAM y un brote de AIJs/Still:

\begin{tabular}{lcc} 
& AIJs & SAM \\
\hline Glóbulos rojos & $\downarrow$ & $\downarrow$ \\
Glóbulos blancos & $\uparrow$ & $\downarrow$ \\
Plaquetas & $\uparrow$ & $\downarrow$ \\
ERS & $\uparrow$ & $\downarrow$ \\
Transaminasas & $\uparrow$ & $\uparrow \uparrow$ \\
Fibrinógeno & $\uparrow$ & $\downarrow$ \\
Triglicéridos & $\mathrm{N}$ & $\uparrow$ \\
Alt. coagulación & - & + \\
Ferritina & $\uparrow$ & $\uparrow \uparrow$ \\
& &
\end{tabular}

Recientemente se han propuesto nuevos criterios diagnóstico para SAM en AIJs ${ }^{30}$.

\section{Síndrome de activación macrofágica}

Paciente febril con AIJs conocida o sospechada ferritina $>684 \mathrm{ng} / \mathrm{mL}$ $+$

al menos dos de los siguientes: Plaquetas $\leq 181 \times 109 / \mathrm{L}$

Transaminasa glutámico oxalacética $>48 \mathrm{U} / \mathrm{L}$ Triglicéridos $>156 \mathrm{mg} / \mathrm{dL}$ Fibrinógeno $\leq 360 \mathrm{mg} / \mathrm{dL}$ 


\section{Púrpura de Schönlein Henöch (PSH) / Vasculitis por IgA}

La PSH afecta predominantemente piel, articulaciones, tracto gastrointestinal y riñones. De curso habitualmente benigno y autolimitado, puede presentar complicaciones que requieren rápida resolución.

El compromiso abdominal se encuentra hasta en el $70 \%$ de los niños, tratándose en la mayoría de ellos de dolor cólico difuso o periumbilical. La complicación más común es la hemorragia del tubo digestivo (hematemesis, melena, hemorragia masiva), siguiéndole en frecuencia la invaginación, más raramente perforación intestinal. Muchas veces la enfermedad debuta con estos síntomas semejando un abdomen agudo, lo que hace difícil el diagnóstico. La ecografía es el método de estudio inicial en estos casos; se puede observar dilatación intestinal, engrosamiento de la pared intestinal, invaginación del intestino delgado (ileo-ileal en la mayoría de los casos) ${ }^{31}$.

Otra complicación aguda más rara de la PSH $(0,8 \%$ a $5 \%)$ es la hemorragia pulmonar ${ }^{32}$. Aparece después del inicio del cuadro purpúrico y se manifiesta por hemoptisis, anemia aguda, infiltrados torácicos, asociándose a alta morbimortalidad.

\section{Enfermedad de Kawasaki (EK)}

La EK es una vasculitis de vasos medianos que requiere de un pronto diagnóstico, ya que la prevención de complicaciones depende de la rápida institución del tratamiento adecuado. Predomina en menores de 5 años y pueden presentarse aneurismas coronarios en el $25 \%$ de los niños no tratados.

Los criterios diagnósticos requieren la presencia de fiebre de 5 o más días de duración, más 4 de los siguientes síntomas principales ${ }^{33}$ :

- Alteraciones en las extremidades: eritema palmar o plantar, edema indurado de manos o pies, descamación periungueal de los dedos

- Exantema polimorfo

- Inyección conjuntival bilateral

- Alteraciones en labios o mucosa oral: labios secos, rojos y fisurados verticalmente, lengua aframbuesada, eritema difuso de la orofaringe

- Adenomegalia cervical unilateral

El compromiso cardiovascular es el que marca el pronóstico de la enfermedad. En la fase aguda puede haber compromiso del endo, mio y/o pericardio. La miocarditis por lo general es leve y transitoria, pero ocasionalmente puede provocar arritmias severas o falla cardíaca aguda.

La afectación coronaria ocurre en el $25 \%$ de los niños no tratados, se desarrolla habitualmente después de los 7-10 días de evolución, aunque puede ser detectada en períodos tempranos. En los casos que presenten afectación coronaria severa puede desarrollarse infarto agudo de miocardio.

\section{Dermatomiositis juvenil (DMJ)}

La DMJ es una enfermedad inflamatoria de origen autoinmune que comienza antes de los 16 años de edad y que afecta predominantemente la piel y músculo esquelético. Se caracteriza por debilidad muscular proximal simétrica, elevación de las enzimas musculares y lesiones cutáneas patognomónicas que son el eritema en heliotropo y las pápulas de Gottron. Habitualmente comienza en forma insidiosa, lo que requiere un alto índice de sospecha para el diagnóstico temprano; en un tercio de los casos el inicio de la enfermedad es agudo y es un claro caso de emergencia en reumatología pediátrica ${ }^{34,35}$.

Una de las complicaciones más graves, con un alto índice de mortalidad, es la vasculitis gastrointestinal, que puede ocasionar sangrado, perforación, infarto mesentérico y neumatosis intestinal. Otra complicación importante que debe evaluarse sistemáticamente, aun en ausencia de síntomas, es la afectación de los músculos del paladar, faringe y porción proximal del esófago; en caso de estar presente debe colocarse sonda nasogástrica para evitar la aspiración pulmonar.

Recientemente fueron publicadas una serie de recomendaciones para el manejo de la DMJ, y la primera recomendación es definir qué pacientes deben ser considerados de alto riesgo para su derivación inmediata a un centro especializado ${ }^{34}$.

Pacientes de alto riesgo:

A. Discapacidad severa, definida por la incapacidad de levantarse de la cama

B. Puntuación CMAS $<15$, o puntuación MMT $8<30$

C. Presencia de aspiración o disfagia

D. Vasculitis gastrointestinal

E. Miocarditis

F. Enfermedad pulmonar parenquimatosa

G. Enfermedad del sistema nervioso central

H. Ulceración de la piel

I. Requerimientos de internación en unidad de cuidados intensivos

J. Edad $<1$ año

\section{Fiebre reumática (FR)}

Es una enfermedad inflamatoria multisistémica desencadenada por una infección por estreptococo $\beta$ hemolítico grupo A (EßHGA) luego de un período de latencia de 2 a 3 semanas. A pesar que su incidencia ha ido disminuyendo en las últimas décadas; la enfermedad cardíaca reumática es la enfermedad cardiovascular adquirida más común en todo el mundo en los menores de 25 años.

No constituye una emergencia médica, salvo excepciones, pero sí una urgencia para efectuar rápida y correctamente el diagnóstico, instituir el tratamiento y evitar el daño cardíaco.

El 75\% de los pacientes presentan una poliartritis migratoria que involucra grandes articulaciones. En algunos pacientes puede ser aditiva o simultánea, afectando en ocasiones pequeñas articulaciones. Su comienzo es agudo presentando los signos clásicos de inflamación y dolor muy intenso. La duración suele ser de 2 a 7 días en cada articulación, y no más de un mes en total. Una característica importante de la artritis es que responde rápidamente al tratamiento con ácido acetilsalicílico.

La carditis se presenta en el $50 \%$ a $75 \%$ de los pacientes. La endocarditis es la localización usual, siendo la válvula mitral la más frecuentemente afectada; le sigue en frecuencia la valvulopatía aórtica que por lo general acompaña a la enfermedad mitral. La miocarditis aislada es rara, lo mismo que la pericarditis; de hecho la presencia de pericarditis en ausencia de valvulopatía debe hacer pensar en otra etiología. El diagnóstico de carditis sigue siendo fundamentalmente clínico, completándose con radiología, electrocardiograma y ecocardiograma.

La gravedad es muy variable, desde afectación subclínica leve a carditis grave con insuficiencia cardíaca congestiva.

El diagnóstico de FR sigue siendo clínico, siendo los criterios de Jones recientemente modificados, los utilizados habitualmente para ello ${ }^{36}$.

\section{Resumen y conclusiones}

- Es un error pensar que no existen o son poco frecuentes las Emergencias/Urgencias en la Reumatología Clínica del adulto y del niño.

- Son muchas las reconocidas y de lo más variadas en tipo y gravedad.

- Debe tenerse un conocimiento amplio de la especialidad que incluye los tratamientos y estar atento a ello.

- La correcta historia clínica del paciente nos permitirá realizar diagnósticos tempranos, de la misma manera tener conocimiento del exacto tratamiento que recibe en la actualidad.

- No menospreciar ningún signo ni síntoma por mínimo que parezca.

- Utilizar los métodos complementarios en forma rápida y 
criteriosa.

- Evaluar con sumo cuidado la utilización de AINE y corticoides en dosis altas, inmunosupresores o terapias biológicas; recordar que las infecciones pueden mimetizar cualquier signo o síntoma de Enfermedad Autoinmune Sistémica.

- A nuestro criterio, la subespecialización condiciona los conocimientos necesarios para una medicina completa y racional en la práctica de la Reumatología Clínica.

\section{BIBLIOGRAFÍA}

1. Armengol G, José J. Urgencias y emergencias médicas. Emergencias. 2014;26.

2. Vymetal J, Skacelova M, Smrzova A, Klicova A, Schubertova M, Horak P, et al. Emergency situations in rheumatology with a focus on systemic autoimmune diseases. Biomed Pap Med Fac Univ Palacky Olomouc Czech Repub. 2016 Mar; 160(1):20-29.

3. Luis Arturo Gutiérrez-González. Review article. Rheumatologic emergencies. Clin Rheumatol. 2015;34:2011-2019.

4. Bella M, Molinari R, Castello L, Sola D, Gibbin A, Guaschino $G$, et al. Profiling the patients visiting the emergency room for musculoskeletal complaints: characteristics and outcomes. Clin Rheumatol. 2016;35:2835-2839.

5. Astetea CG, Kaumib L, Tejada Sorados RM, Medina Quinones C, Borja Serrati JF. Prevalencia de la afección musculoesquelética no traumática como motivo de consulta y su impacto asistencial en un servicio de urgencias. Semergen, Medicina de familia. 2016;42(3):158-163.

6. Guillén-Astetea CA, Boteanua A, Blázquez-Canameroa MA, Villarejo-Botija M. La consulta especializada de reumatología en un servicio de urgencias: un año de experiencia con la unidad de urgencias reumatológicas y musculoesqueléticas (URMES). Reumatol Clin. 2017;13(1):21-24.

7. Guillen Astete C, Medina Quinones C, Borja Serrati J, Redondo Romero C, Ahijón Lana M, Anton Pages F, et al. ¿Cuál puede ser el papel de la Reumatología en un Servicio de Urgencias? Reumatol Clin. 2012;8:33-4.

8. Arturi AS. Guías clínicas para el estudio y diagnóstico de enfermedades en Reumatología. Argentina: Ed. Abbvie SA; 2010;49-125.

9. Arturi AS. Diagnóstico en reumatología y enfermedades autoinmunes sistémicas. Argentina: Ed. Abbvie SA; 2014;cap:6,9,10,14.

10. Arturi AS. Marcos JC, BabiniJC. Enfermedades autoinmunes. Argentina: Ed. Abbott SA; 2004;39-111.

11. Alva Feriz R. Muñoz Louis R, Espinosa LR, Kamashta M, Matucci Cerini M, Sanmarti M. Enfermedades inflamatorias en Reumatología. Ed. PANLAR; 2016;caps:11,12,14,16,18,19.2 $1,23,24$.

12. Sociedad Española de Reumatología. Manual SER de diagnóstico y tratamiento de las enfermedades reumáticas autoinmunes sistémicas. España: Elsevier; 2014;99-297.

13. Martinez-Martinez MU, Abud-Mendoza C. Diffuse alveolar hemorrhage in patients with systemic lupus erythematosus. Clinical manifestations, treatment, and prognosis. Reumatol Clin. 2014;10(4):248-53.

14. Bertsias GK, Ioannidis JP, Aringer M, Bollen E, Bombardieri $\mathrm{S}$, BruceI $\mathrm{N}$, et al. EULAR recommendations for the management of systemic lupus erythematosus with neuropsychiatric manifestations: report of a task force of the EULAR standing committee for clinical affairs. Ann Rheum Dis. 2010;69(12):2074-82.

15. Keeling D, Mackie I, Moore GW. British Committee for Standards in Haematology. Br J Haematol. 2012;157(1):47-58.

16. West SC, Arulkumaran N, Philip W, Pusey CD. Pulmonaryrenal syndrome: a life threatening but treatable condition. Postgrad Med J. 2013;89:274-283.

17. Nayer A, Ortega LM. Guidelines on the investigation and management of antiphospholipid syndrome. Catastrophic antiphospholipid syndrome: a clinical review. J Nephropathol. 2014;3(1):9-17.

18. Fialho SC, de Castro GR, Zimmermann AF, Ribeiro GG, Neves FS, Pereira IA, et al. Musculoskeletal system assessment in an emergency room. Rev Bras Reumatol. 2011;51:240-8.

19. Lee RW, D'Cruz DP. Pulmonary renal vasculitis syndromes. Autoimmun Rev. 2010; 9:657-660.

20. Marcos JC, Arturi AS, Babini JC. GOTA. Ed. G/B Editores; 1995;cap:6.

21. Terkeltaub R. Gout and other crystal arthropathies. Ed. Elsevier; 2012;cap:8.

22. Muñoz-Egea MC, Blanco A, Fernández-Roblas R, Gadea I, García-Cañete J, Sandoval E, et al. Clinical and microbiological characteristics of patients with septic arthritis: a hospital-based study. J Orthop. 2014;11:87-90.

23. Southerland LT, Richardson DS, Caterino JM, Essenmacher AC, Swor RA. Emergency department recidivism in adults older than 65 years treated for fractures. Am J Emerg Med. 2014;32:1089-92.

24. Friedman BW, Chilstrom M, Bijur PE, Gallagher EJ. Diagnostic testing and treatment of low back pain in United States emergency departments: a national perspective. Spine. 2010;35: E1406-11.

25. Thiruganasambandamoorthy V, Turko E, Ansell D, Vaidyanathan A, Wells GA, Stiell IG. Risk factors for serious underlying pathology in adult emergency department nontraumatic low back pain patients. J Emerg Med. 2014; 47:1-11.

26. Shenoi S, Wallace CA. Diagnosis and Treatment of Systemic Juvenile Idiopathic Arthritis.J Pediatr. 2016 Oct;177:19-26.

27. Inoue N, Shimizu M, Tsunoda S, Kawano M, Matsumura M, Yachie A. Cytokine profile in adult-onset Still's disease: Comparison with systemic juvenile idiopathic arthritis. Clin Immunol. 2016 Aug;169:8-13.

28. Yamaguchi $\mathrm{M}$, Ohta A, Tsunematsu $\mathrm{T}$, Kasukawa $\mathrm{R}$, Mizushima $\mathrm{Y}$, Kashiwagi $\mathrm{H}$, et al. Preliminary criteria for classification of adult Still's disease. J Rheumatol. 1992;19:424-430.

29. Reddy V, Myles A, Cheekatla S, Singh S, Aggarwal A. Soluble CD25 in serum: a potential marker for subclinical macrophage activation syndrome in patients with active systemic onset juvenile idiopathic arthritis. International Journal of Rheumatic Diseases. 2014;17:261-267.

30. Angelo Ravelli, et al. 2016 Classification Criteria for Macrophage Activation Syndrome Complicating Systemic Juvenile Idiopathic Arthritis. Arthritis Rheumatol. 2016 Mar;68(3):566-76.

31. Lerkvaleekul B, Treepongkaruna $S$, Saisawat $P$, Thanachatchairattana $\mathrm{P}$ Angkathunyakul N, Ruangwattanapaisarn N, Vilaiyuk S. Henoch-Schönlein purpura from vasculitis to intestinal perforation: A case report and literature review. World J Gastroenterol. 2016 July 14;22(26):6089-6094.

32. Rajagopala S, Shobha V, Devaraj U, D'Souza G, Garg I. Pulmonary hemorrhage in Henoch-Schönlein purpura: case report and systematic review of the english literature. Semin Arthritis Rheum. 2013 Feb;42(4):391-400.

33. McCrindle BW, Rowley AH, Newburger JW, Burns JC, Bolger AF, Gewitz M, et al. Diagnosis, Treatment, and Long-Term Management of Kawasaki Disease: A Scientific Statement for Health Professionals From the American Heart Association. Circulation. 2017 Apr 25;135(17):927-999.

34. Enders FB, Bader-Meunier B, Baildam E, Constantin $\mathrm{T}$, Dolezalova $\mathrm{P}$, Feldman BM, et al. Consensus-based recommendations for the management of juvenile dermatomyositis. Ann Rheum Dis. 2017;76:329-340.

35. Singh S, Suri D, Aulakh R, Gupta A, Rawat A, Kumar RM. Mortality in children with juvenile dermatomyositis: two decades of experience from a single tertiary care centre in North India. Clin Rheumatol. 2014; 33:1675-1679.

36. Gewitz MH, Baltimore RS, Tani LY, et al. Revision of the Jones criteria for the diagnosis of acute rheumatic Fever in the era of Doppler echocardiography: a scientific statement from the American heart association. Circulation. 2015;131:1806-18. 